\title{
Management of Pseudotumor Cerebri Secondary to Dural Venous Sinus Thrombosis in Children
}

\author{
Mohamed M Elsherbini ${ }^{1}$, Hatem Badr ${ }^{1}$, Mohamed Kassem ${ }^{1}$, Amr Farid Khalil ${ }^{1}$
}

\author{
${ }^{1}$ Neurosurgery Department, Mansoura \\ University Hospital, Mansoura \\ University, Mansoura, Egypt.
}

To whom correspondence should be addressed: Mohamed M Elsherbini, RN

e-mail:

m_elsherbiny@mans.edu.eg

Available at:

http://www.archpedneurosurg. com.br/

\begin{abstract}
Background: The purpose of this study is to follow the clinical course and required management of children suffering from Pseudotumor cerebri (PTC) secondary to Dural Sinus Thrombosis (DST) to understand the natural history of a rare condition and its need for surgery as well as outcome after Cerebro-Spinal Fluid (CSF) diversion surgeries.

Methods: A retrospective consecutive case series study based on examining medical records of patients who were referred to neurosurgical pediatric clinic suffering from PTC secondary to DTS. Data review included patients' archives for clinical, radiological, surgical records and follow up visits. Only patients below 18 years old were included.

Results: Fourteen patients met inclusion criteria, 7 of them required CSF diversion procedure, while the other $50 \%$ responded to medical conservative therapy. Headache improved immediately for most of the surgical group, while took 7 weeks to reach satisfactory results for medical group. Visual improvement took place for both groups approximately at the same time interval with average 5 weeks.

Conclusion: Surgical intervention in the form of CSF diversion procedure was required for $50 \%$ of the patients, which is safe and effective line of treatment to prevent further deterioration of vision for case who were not responsive to medical therapy.
\end{abstract}

Keyworks: Dural sinus thrombosis, Pseudotumor cerebri, Lumboperotineal shunt, Idiopathic Intracranial Hypertension

\section{INTRODUCTION}

Pseudo tumor cerebri (PTC) is a poorly understood pathology characterized by raised intracranial pressure and papilledema without underlying parenchymal lesion, mass or ventriculomegaly according to Monroe-Kellie (1) alimentary rule. Formerly, the term IIH (Idiopathic Intracranial Hypertension) was widely used, however consecutive publications described the same clinical features as sequelae of other pathologies, henceforward the term IIH, or primary PTC, is saved to conditions where a primary cause couldn't be detected. While the term (Benign intracranial hypertension) is totally abandoned due to the possible serious sequelae of the disease e.g. optic atrophy. The most scientifically accepted term is Pseudotumor Cerebri (PTC) (2)
Secondary PTC is mainly described as a sequalae of Dural Sinus Thrombosis (DST) which is rare in pediatric age group with an incidence of less than 1 per 100,000 people annually (3). The majority of these cases present with acute serious manifestations with parenchymal changes which doesn't match the criteria of PTC diagnosis, this makes secondary PTC is limited to cases presented with headache, papilledema and/or cranial nerve palsy which are among the least common presentations of dural sinus thrombosis (DST) (4).

Dural sinus thrombosis in children is a rare pathology with increasing ratio of diagnosed cases in the past decade, this could be attributed to modern imaging modalities, its feasibility and availability. Causes of this pathology among pediatric group are not well understood, in most cases it was 


\section{Management of Pseudotumor Cerebri Secondary to Dural Venous Sinus Thrombosis in Children}

linked to infection or coagulation disorder (5). PTC secondary to dural venous sinus thrombosis in children is a condition with no firmly established treatment guidelines due to the paucity of cases, that's why patients are managed individually according to disease progress for each. There is significant data in literature on dural venous thrombosis sequelae in adults, while much less data found regarding pediatric population. The purpose of this study is to retrospectively follow the course and management of 14 consecutive cases of PTC secondary to DST who were managed in a tertiary referral center.

\section{PATIENTS AND METHODS}

Study design: An observational retrospective consecutive case series study.

Patient selection: Patients presented to neurosurgical pediatric outpatient clinic of Mansoura University Hospital were reviewed for the years 2018 and 2019, patients' archives and medical records were analyzed. Only pediatric group were included (age below 18), patients with at least one dural venous sinus thrombosis were included otherwise excluded. Both patients who improved on medical treatment and those who required surgical intervention were included. Adults and patients with primary PCT were excluded. Patients with dural sinus thrombosis not leading to PTC were not included in this study.

Data extraction: After institutional agreement and consent waiver due to the retrospective nature of the study, patients' records including presentation, course and management of the disease were analyzed, as well as radiology mainly Magnetic Resonance Imaging (MRI) and Magnetic Resonance Venography (MRV). Clinical investigations like Optical Coherence Tomography (OCT) photographs were reviewed in correlation to clinical records.

Treatment protocol: Surgical intervention was indicated as the first line of management on urgent basis for patients who were presented with papilledema grade 4 , followed by anticoagulant therapy 48 hours post-operatively, which is then tapered over 6 months.

Patients with papilledema grades 2 and 3 received anticoagulant therapy after initial Lumbar puncture to confirm diagnosis and measure opening pressure, for this group weekly lumbar puncture was performed after intermittent stoppage of anticoagulant to follow progress of the disease via measuring opening pressure. Patients with vision worsening, progression of papilledema or raised opening pressure were indicated for surgery after 48 hours stoppage of anticoagulant. Carbonic anhydrase inhibitor and diuretic were received all over the course of with potassium supplement and thorough monitoring of serum potassium level. According to our organization protocol, Frisen papilledema grading system (Table 1 ) is adopted, primarily clinically by fundoscopy.

Table 1- Key clinical features of papilledema grading (6)

\begin{tabular}{|c|c|}
\hline $\begin{array}{l}\text { Papilledema } \\
\text { grade }\end{array}$ & Features \\
\hline Grade 0 & Normal optic disc \\
\hline Grade 1 & $\begin{array}{l}\text { Minimal edema: C-shaped halo that is subtle } \\
\text { and grayish with a temporal gap; obscures } \\
\text { underlying retinal details }\end{array}$ \\
\hline Grade 2 & $\begin{array}{l}\text { Mild edema: Circumferential halo, elevation of } \\
\text { nasal border }\end{array}$ \\
\hline Grade 3 & $\begin{array}{l}\text { Moderate edema: Obscuration of more than } 1 \\
\text { segment of major blood vessels leaving disc, } \\
\text { circumferential halo i.e elevation of all borders. }\end{array}$ \\
\hline Grade 4 & $\begin{array}{l}\text { Marked edema: Total obscuration of a } \\
\text { segment of a major blood vessel on the disc, } \\
\text { complete halo }\end{array}$ \\
\hline Grade 5 & $\begin{array}{c}\text { Obscuration of all vessels on the disc and } \\
\text { leaving the disc }\end{array}$ \\
\hline
\end{tabular}

Anticoagulant agents: patients who were managed conservatively received subcutaneous low molecular weight heparin initially to allow intermittent stoppage for lumbar puncture or surgery when indicated, afterwards shifted to oral anticoagulant (Warfarin), while patients who underwent surgery received subcutaneous low molecular weight heparin 48 hours after surgery which is shifted to oral anticoagulant (Warfarin) one week after surgery.

Work up for predisposing factors: included basic investigations; blood picture, renal functions and hepatic enzymes as well as investigations for septic focus and otoscopic examination to confirm or rule out otitis media. Coagulation profile including D-dimer was performed for all patients.

\section{RESULTS}

Fourteen patients were included in the study who met the inclusion criteria, 7 were male. Age median was 12 years which ranged between 7 and 15 years. Headache was among the presenting symptoms for all cases, while blurred vision was mentioned by 12 patients. Abducens nerve palsy was found in 4 patients (28. \%) and only two patients were presented with seizures. Seven cases gave history of fever prior to presentation, range 5 - 13 days, two of them were known cases of recurrent otitis media, while the other five were treated outside the institute with empirical antibiotics, workout was performed upon admission; urine culture, blood culture and coagulation profile were normal. Otoscopic examination showed retraction of tympanic membrane indicating healing otitis media. Two patients showed coagulation disorders identified via elevated Ddimer however the primary cause couldn't be identified, while no predisposing factors could be identified for the rest of patients. 


\section{Management of Pseudotumor Cerebri Secondary to Dural Venous Sinus Thrombosis in Children}

Table 2 - Summary of clinical and radiological features, management and follow up. (SSS: Superior Sagittal Sinus, TS: Transverse Sinus, LP: Lumbo-peritoneal, VP: Ventriculo-peritoneal)

\begin{tabular}{|c|c|c|c|c|c|c|c|c|c|}
\hline \multirow[t]{2}{*}{ Patient } & \multirow{2}{*}{$\begin{array}{l}\text { Age } \\
\text { (years) }\end{array}$} & \multirow[t]{2}{*}{ Sex } & \multirow{2}{*}{$\begin{array}{l}\text { Opening } \\
\text { Pressure } \\
\text { (cm H2O) }\end{array}$} & \multirow{2}{*}{$\begin{array}{l}\text { Papilledema } \\
\text { Grade }\end{array}$} & \multicolumn{3}{|c|}{ MRV and MRI } & \multirow[t]{2}{*}{ Management } & \multirow{2}{*}{$\begin{array}{c}\text { Follow } \\
\text { up } \\
\text { (months }\end{array}$} \\
\hline & & & & & $\begin{array}{l}\text { Venous } \\
\text { Sinus } \\
\text { thrombosis }\end{array}$ & Empty sella & $\begin{array}{l}\text { tortuous } \\
\text { optic } \\
\text { nerve }\end{array}$ & & \\
\hline 1 & 13 & $M$ & 40 & 4th & SSS & & & LP shunt & 6 \\
\hline 2 & 11 & $\mathrm{~F}$ & 36 & $3 r d$ & TS & & V & LP shunt & 9 \\
\hline 3 & 15 & $\mathrm{~F}$ & 34 & $3 r d$ & TS & V & $\mathrm{V}$ & Conservative & 12 \\
\hline 4 & 12 & $F$ & 33 & 2nd & TS & & & Conservative & 8 \\
\hline 5 & 11 & $\mathrm{~F}$ & 30 & 2nd & TS & & & Conservative & 9 \\
\hline 6 & 12 & $\mathrm{~F}$ & 32 & $2 n d$ & TS & & & Conservative & 12 \\
\hline 7 & 12 & $\mathrm{M}$ & 40 & 4th & TS & & V & LP shunt & 12 \\
\hline 8 & 11 & $\mathrm{~F}$ & 35 & $3 r d$ & TS & & & Conservative & 12 \\
\hline 9 & 13 & $M$ & 33 & $2 n d$ & TS & & & LP shunt & 9 \\
\hline 10 & 8 & $\mathrm{M}$ & 40 & $3 r d$ & TS & & & VP shunt & 12 \\
\hline 11 & 7 & $\mathrm{M}$ & 33 & $3 r d$ & TS & & & Conservative & 9 \\
\hline 12 & 12 & $M$ & 37 & $2 n d$ & TS & & & VP shunt & 9 \\
\hline 13 & 10 & $M$ & 42 & $3 r d$ & TS & & & Conservative & 6 \\
\hline 14 & 15 & $\mathrm{~F}$ & 36 & $3 r d$ & TS & & & LP shunt & 12 \\
\hline
\end{tabular}

Opening pressure was elevated in all cases, with median of $35.5 \mathrm{~cm} \mathrm{H} 2 \mathrm{O}$, which ranged from 30 to $42 \mathrm{~cm} \mathrm{H} 2 \mathrm{O}$. Papilledema as a cardinal sign was noticed in all cases with different grades ranging from grade 2 in 5 patients, grade 3 in 7 patients and grade 4 in 2 patients. Predisposing factors to sinus thrombosis were identified in 9 cases; 7 otitis media and 2 coagulation disorders (Table 2 ).

Radiologically one patient suffered PCT secondary to superior sagittal sinus thrombosis while transverse sinus thrombosis was found in the rest (13 patients), one patient's radiology showed empty sella syndrome and 3 patients (21.4 \%) showed tortious optic nerve which is a radiological sign of raised intracranial pressure and one of the radiological findings of PTC (7)

Seven patients (50\%) underwent Cerebrospinal Fluid (CSF) diversion surgery (5 Lumbo-peritoneal (LP) shunts and 2 Ventriculo-peritoneal (VP) shunts), while 7 patients improved on anti-coagulant therapy. Two patients showed papilledema grade 4 at presentation requiring surgical intervention without initial anticoagulant therapy. The other five patients of the surgical group, two grade 2 and three grade 3 papilledema, were indicated for surgery after failure of medical therapy and progression of clinical findings. For the latter group medical therapy ranged from 7 to 21 days (mean 12 days) before being indicated for surgery.
Patients follow up continued up to one year with minimal follow up of 6 months with mean follow up period of 9.8 months. One patient who underwent LP shunt was complicated with infection and removal later.

Patients who were managed medically complained of residual headache for variable durations ( 1 to 7 weeks), on the other hand 4 patients (57\%) of the surgical group showed immediate post-operative relief of headache, the other 3 patients improved over four weeks post-operatively. All patients showed improved vision during follow up with heterogenous time intervals, with average 5 weeks to return to normal for both groups. None of the patients of the study was suspected to be atretic rather than thrombosed sinus based on clinical and radiological findings.

\section{ILLUSTRATIVE CASES}

Two cases were selected to illustrate both medical and surgical courses of management, one of each group.

Case 1

Eleven years old girl, presented to pediatric neurological clinic with headache, blurred vision and sudden right 6th nerve palsy, MRI brain showed no parenchymal lesions, MRV showed left transverse sinus dropout with normal brain parenchyma in FLAIR MRI (Fig 1), atretic transverse sinus was excluded by $\mathrm{CT}$ bone window showing prominent sigmoid 


\section{Management of Pseudotumor Cerebri Secondary to Dural Venous Sinus Thrombosis in Children}

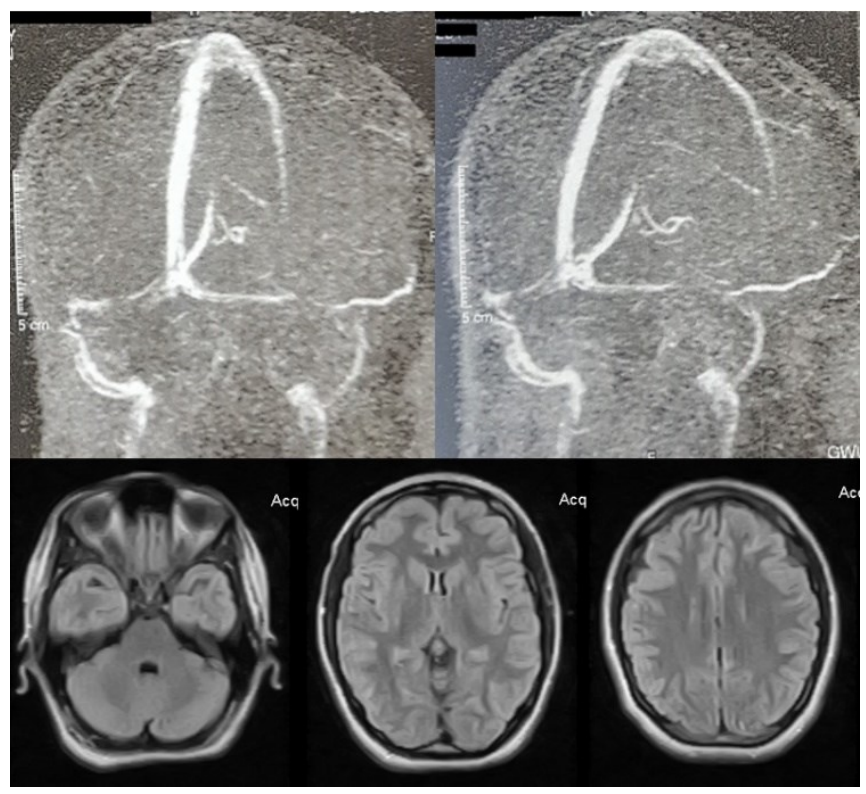

Fig 1- MRV and MRI brain of 11 years old girl, showing thrombosed left transverse sinus, normal brain parenchyma.

notch on the left side. On examination, patient was fully conscious, papilledema grade 2 and opening pressure $30 \mathrm{~cm}$ $\mathrm{H} 2 \mathrm{O}$. Patient was treated for the first three days with low molecular weight Heparin, followed by Warfarin with adjusted INR to 2. Patient was followed for clinical improvement especially papilledema, the patient course was improving till complete resolution of symptoms and signs by the third week. Follow up period continued to 9 months with no recurrence of symptoms.

\section{Case 2}

Twelve years old boy, presented with headache and rapid deterioration of vision over few days, on examination patient was neurologically intact except for papilledema grade 4 while opening pressure was $40 \mathrm{~cm} \mathrm{H2O}, \mathrm{MRI}$ showed tortious optic (Fig 2) nerve and MRV showed obliteration of transverse sinus on the right side with normal brain parenchyma in FLAIR MRI (Fig 3), atretic sinus was excluded by CT brain and bone window as the sigmoid sinus notch was prominent bilaterally (Fig 4). The clinical presentation was alarming and indicated urgent surgical interference with LP shunt, followed by anticoagulant therapy after safe time interval. Patient was followed for clinical improvement, after 3 weeks patient's fundus examination was free. Follow up period lasted 12 months with no recurrence of symptoms.

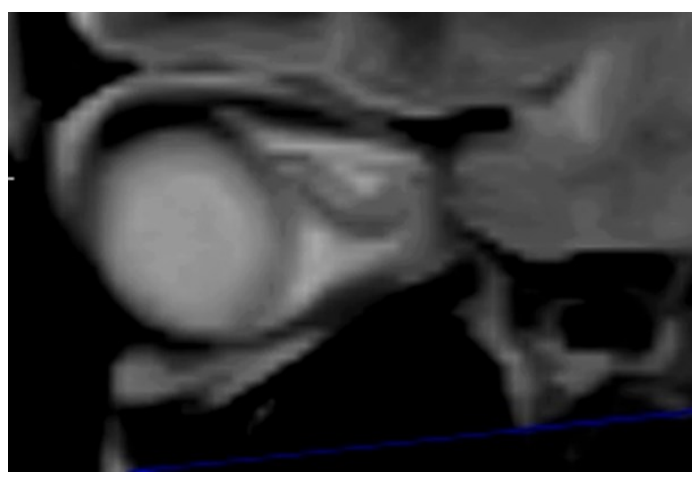

Fig 2 - Tortuosity of optic nerve.

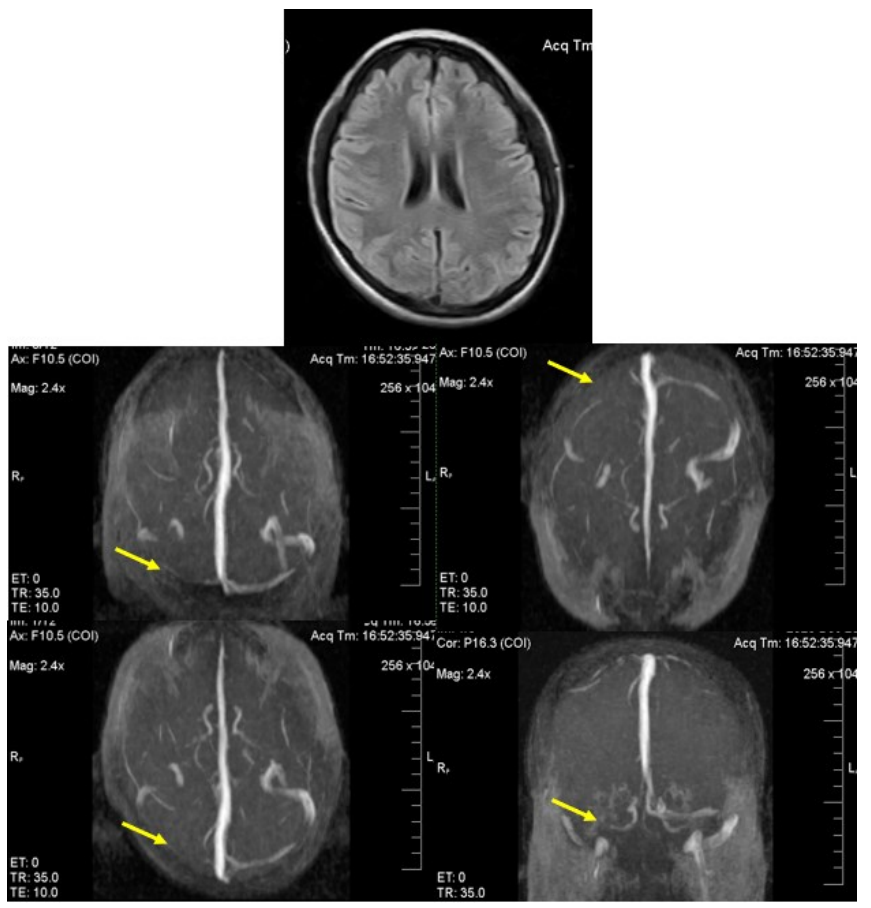

Fig 3 - MRV and MRI brain of 12 years old boy showing right transverse sinus thrombosis (yellow arrows), normal brain parenchyma.

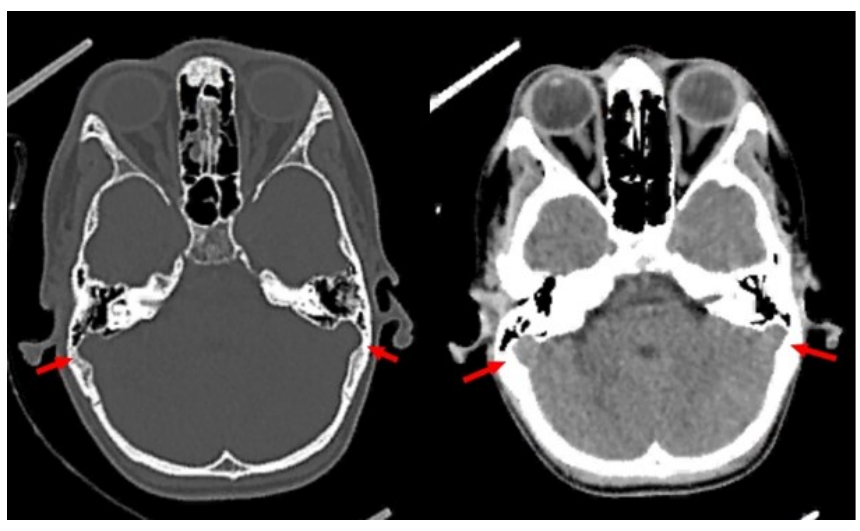

Fig 4 - Negative sigmoid sinus notch sign i.e. symmetry of the notch bilaterally ruling out atresia (red arrows) 


\section{Management of Pseudotumor Cerebri Secondary to Dural Venous Sinus Thrombosis in Children}

\section{DISCUSSION}

PTC in children is more likely to be secondary than idiopathic (55\%). In spite of its rarity in pediatric population, dural venous sinus thrombosis accounts for the majority of pediatric PTC, other causes are reported like endocrinal abnormalities, venous anomalies and drugs (8). Although DST is more common in the first 6 months of life than older children, no PTC cases were reported in literature in this age as well as our study, which could be explained by the compliance of brain at this age (9). Female predominance was reported with primary $\mathrm{IIH}$, while secondary cases were not gender linked, in this study male to female ratio was 1:1.

Diagnosis of PTC in children is difficult as the main two presenting symptoms; headache and deterioration of vision are subjective and difficult to be expressed by younger children. Headache is the presenting symptom of DST in up to $91 \%$ of children (10), theoretically this percent is expected to be higher when complicated with PTC. In our study's age group ( 8 to 15 years), patients were capable to demonstrate their complaints where headache was the presenting symptom in all patients.

According to the revised criteria of Friedman for PTC diagnosis, this study included 14 cases with definitive diagnosis, considering the revised upper limit of normal OP in pediatric group as $28 \mathrm{~cm} \mathrm{H} 2 \mathrm{O}$. All patients were presented with headache and most of the cases $(85.7 \%)$ were presented with visual complaints. Abducent nerve palsy is a common complication of raised intracranial pressure (ICP) which was present in (28.6\%) of our patients. Papilledema is the hallmark clinical sign, however PTC without papilledema is still a probable diagnosis, a condition not encountered in this study (4). OCT is a non-invasive method to visualize the optic nerve that has been used to diagnose and follow the course of the disease, in our study it matched the clinical fundus examination (11). Disturbance of consciousness and/or neurological deficits, which are typically absent in both PTC and DST, were not encountered in this study.

MRI is essential for PTC diagnosis to exclude parenchymal lesions and to identify radiological signs of raised ICP; flattening of the globe, tortious optic nerve and empty sella (12). In our series radiological signs of raised ICP were not significantly linked to the diagnosis, only three patients showed tortious optic nerve with one patient of empty sella as well. MRV is the cornerstone to diagnose sinus thrombosis in children which was the diagnostic tool in all our patients. Differentiation between thrombosed and atretic dural venous sinus is an important step to reach proper diagnosis, in this study the sigmoid sinus notch sign (Fig 6) is adopted to rule out atretic transverse sinus, as well as unmistakable clinical features. Sigmoid sinus notch sign depends on the symmetry of the sigmoid sinus notch bilaterally in noncontrast CT scan, as it's expected that the bony notch to be shallower on the side of the atretic transverse sinus, this sign when combined with MRV gives accurate differentiation between thrombosis and atresia of the transverse sinus (13).

Predisposing factors for DST include a wide variety of local and systemic pathologies e.g. nephrotic syndrome, infections, systemic lupus, otitis media, mastoiditis, sinusitis, trauma, dehydration, anemia, leukemia, sepsis and chemotherapy (14). Though predisposing factors are identifiable for $90 \%$ of cases according to multicentric cohort study (9), in this case series we were able to identify risk factors for only $64.2 \%$ of patients $(9 / 14)$, this could be attributed to prior management of those patients outside the institute where empirical use of broad spectrum antibiotics is a common practice.

In this study, 13 patients (92.9\%) were diagnosed with transverse sinus thrombosis, which is, as well, the most common in registry with a rate of $73 \%$. In line with literature (8), obesity was not an associated risk factor.

To our knowledge, mortality due to secondary PTC in children are not estimated in literature, while dural sinus thrombosis carries the risk of $13 \%$ mortality in children with higher percent $(67 \%)$ in neonates (9) , this could explain that no mortalities were reported in this study, since our case series didn't include any neonates.

Mild cases of DSTcan be managed conservatively with hydration and serial ophthalmological examinations, papilledema is a red flag to start aggressive therapy. Conservative therapy, in our study, aimed at decreasing ICP via carbonic anhydrase inhibitors and diuretics while patient is on low molecular weight heparin accompanied by repeated lumbar tap with intermittent interruption of anticoagulant therapy (15). According to European Pediatric Neurology Society and French Society for Pediatric Neurology (EPNS/SFNP) guidelines, anti-coagulant therapy is considered safe without increased risk of intracranial hemorrhage with no evidence of superiority of a certain agent (16).

Papilledema grade is not only strongly linked to the severity of the pathology (17), but also an important predictor to visual recovery or deterioration (18). The traditionally adopted Frisen grading system classified papilledema according to optic disc oedema and bulge into six grades, where grade 0 represents normal fundus while grade 5 shows obscuration of all vessels on the disc and leaving the disc i.e. pre-atrophy stage (6). No cases with papilledema grade 5 were encountered in this series, which could be attributed to progressive course of symptoms leading to seeking medical advice and eventual management. In this series papilledema and OP were followed during anti-coagulant therapy, medical therapy alone was sufficient for $50 \%$ of cases, while $50 \%$ required CSF 


\section{Management of Pseudotumor Cerebri Secondary to Dural Venous Sinus Thrombosis in Children}

diversion procedure, 3 patients who were presented with papilledema grade 4 underwent CSF diversion directly on urgent basis, while the other four $(28.9 \%)$ patients were indicated for surgery after failed medical therapy. Though hydration and antibiotic proved effective in $70 \%$ of children with uncomplicated dural sinus thrombosis (9), patients with PTC on top of DST were not subjective to this routine due to possibility of visual loss over long treatment plan.

Surgical management is indicated after failure of medical therapy (19) and/or in emergent cases who are presented with high papilledema grade,impending visual loss due to post-papilledema visual atrophy is the most feared complication. Abrupt increase of opening pressure indicates rapid surgical intervention, while slow course allows the compliance of brain parenchyma, intracranial blood and CSF to adapt which gives chance for conservative therapy. Surgical management includes optic nerve fenestration which was not conducted to any of our patients and CSF diversion procedures which remain the safest and most used surgical intervention. LP shunt has been globally used and studied; VP shunt is recently introduced especially after the wide use of neuro-navigation to target the slit ventricle with least complications, the rationale of VP shunt in such cases is to avoid tonsillar decent which is reported after lumbar CSF diversion. In our study, most of the surgical group (5/7) patients underwent LP shunt surgery based on surgeons' preference and family counselling, no cases showed acquired Chiari malformation during the follow up period.

Limitations of study includes the few number of cases and heterogeneity of surgical treatment between LP and VP shunts, a prospective randomized study is encouraged to better define the indications for each procedure. Also, long term follow-up of these patients to detect tonsillar herniation is needed.

\section{CONCLUSION}

PTC secondary to DST is a disabling disease in children which, if not properly managed, can lead to visual loss. CSF diversion, when indicated, is safe and effective intervention not only to prevent further visual impairment and subsequently blindness but also to cure deteriorated visual functions especially for patients presented with high grade papilledema and it's indicated in $50 \%$ of cases. Further studies to compare LP shunt and VP shunt are encouraged to identify timeline for improvement for both groups.

\section{DISCLOSURES}

The authors have no personal, financial, or institutional interest in any of the materials, or devices described in this article.

\section{REFERENCES}

1. Beck TS. on the Structure and Functions of the Sympathetic Nervous System,. As Distinct From, and Independent of, the Cerebro-Spinal System. Lancet. 1847;49(1241):615-7.

2. Daniels $A B$, Liu GT, Volpe NJ, Galetta SL, Moster ML, Newman NJ, et al. Profiles of Obesity, Weight Gain, and Quality of Life in Idiopathic Intracranial Hypertension (Pseudotumor Cerebri). Am J Ophthalmol. 2007;143(4).

3. Shroff M, DeVeber G. Sinovenous thrombosis in children. Vol. 13, Neuroimaging Clinics of North America. 2003. p. 115-38.

4. Friedman DI, Jacobson DM. Diagnostic criteria for idiopathic intracranial hypertension. Vol. 59, Neurology. 2002. p. 1492-5.

5. Gilbert AL, Heidary G. Update on the evaluation of pediatric idiopathic intracranial hypertension. Vol. 27, Current Opinion in Ophthalmology. 2016. p. 493-7.

6. Scott CJ, Kardon RH, Lee AG, Frisén L, Wall M. Diagnosis and grading of papilledema in patients with raised intracranial pressure using optical coherence tomography vs clinical expert assessment using a clinical staging scale. Arch Ophthalmol. 2010;128(6):705-11.

7. Bidot S, Saindane AM, Peragallo JH, Bruce BB, Newman NJ, Biousse V. Brain Imaging in Idiopathic Intracranial Hypertension. Journal of Neuro-Ophthalmology. 2015.

8. Rogers DL. A review of pediatric idiopathic intracranial hypertension. Vol. 61, Pediatric Clinics of North America. 2014. p. 579-90.

9. Wasay M, Dai Al, Ansari M, Shaikh Z, Roach ES. Cerebral venous sinus thrombosis in children: A multicenter cohort from the United States. J Child Neurol. 2008;23(1):26-31.

10. Aylward SC, Waslo CS, Au JN, Tanne E. Manifestations of Pediatric Intracranial Hypertension From the Intracranial Hypertension Registry. Pediatr Neurol. 2016;61:76-82.

11. Avery RA, Licht DJ, Shah SS, Huh JW, Seiden JA, Boswinkel J, et al. CSF opening pressure in children with optic nerve head edema. Neurology. 2011;76(19):165861.

12. Suzuki H, Takanashi J, Nagasawa K, Kobayashi K, Tomita $M$, Tamai $K$, et al. Clinical and MRI findings in childhood idiopathic intracranial hypertension. No To Hattatsu. 2001;33(4):319-22.

13. Chik Y, Gottesman RF, Zeiler SR, Rosenberg J, Llinas RH. Differentiation of transverse sinus thrombosis from congenitally atretic cerebral transverse sinus with CT. Stroke. 2012;43(7):1968-70.

14. Dlamini N, Billinghurst L, Kirkham FJ. Cerebral Venous Sinus (Sinovenous) Thrombosis in Children. Vol. 21, Neurosurgery Clinics of North America. 2010. p. 511-27. 


\section{Management of Pseudotumor Cerebri Secondary to Dural Venous Sinus Thrombosis in Children}

15. Fitzgerald KC, Williams LS, Garg BP, Carvalho KS, Golomb MR. Cerebral sinovenous thrombosis in the neonate. Arch Neurol. 2006;63(3):405-9.

16. Johnson MC, Parkerson N, Ward S, De Alarcon PA. Pediatric sinovenous thrombosis. J Pediatr Hematol Oncol. 2003;25(4):312-5.

17. Tang L, Kardon RH, Wang JK, Garvin MK, Lee K, Abràmoff MD. Quantitative evaluation of papilledema from stereoscopic color fundus photographs. Investig Ophthalmol Vis Sci. 2012;53(8):4490-7.
18. Micieli JA, Bruce BB, Vasseneix C, Blanch RJ, Berezovsky $\mathrm{DE}$, Newman NJ, et al. Influence of Optic Nerve Appearance on Visual Outcome in Pediatric Idiopathic Intracranial Hypertension. Can J Neurol Sci. 2020;47(5):661-5.

19. Julayanont $P$, Karukote $A$, Ruthirago D, Panikkath D, Panikkath R. Idiopathic intracranial hypertension: Ongoing clinical challenges and future prospects. Vol. 9, Journal of Pain Research. 2016. p. 87-99. 